\title{
ECG Signal Denoising and Ischemic Event Feature Extraction using Daubechies Wavelets
}

\author{
H.S.Niranjana Murthy \\ M.S.Ramaiah Institute of Technology \\ Bangalore-54 \\ India
}

\author{
M.Meenakshi, PhD. \\ Dr.Ambedkar Institute of Technology \\ Bangalore-56 \\ India
}

\begin{abstract}
The ability of an intelligent system to correctly classify and recognize patterns makes them particularly suitable for use in an expert system that aids in the interpretation of ECG signals. The features extracted from ECG are highly useful in diagnosis. Wavelet based methods present a best performance as irregularity measures and makes them suitable for ECG data analysis. In this paper, we propose an algorithm for detection of myocardial Ischemic episodes from Electrocardiogram (ECG) signal using Daubechies Wavelet transform technique. The ECG signal was denoised by removing the corresponding wavelet coefficients at higher scale. Analysis is carried out using MATLAB software. The algorithm was evaluated using two cases of data, the first case is with healthy subjects, and second case is with subjects affected by myocardial Ischemia. ECGs are obtained from MIT-BIH Arrhythmia Database which is manually annotated and developed for validation. From the results, it is concluded that Daubechies wavelets are best suitable for small datasets and are able to clearly demark the healthy and disease subjects such as myocardial ischemia subjects.
\end{abstract}

\section{General Terms}

Feature extraction, classification, arrhythmias,

\section{Keywords}

Daubechies wavelets, Myocardial Ischemia, Feature extraction

\section{INTRODUCTION}

Electrocardiography deals with the electrical activity of the heart. The electrocardiogram (ECG) is a record of the origin and propagation of electrical potential through cardiac muscles and examined by placing sensors at limb extremities of the subject. It is considered a representative signal of cardiac physiology, useful in diagnosing cardiac disorders [2].

The cardiac health status is determined by the shape of the Electrocardiogram, which contains important pointers to different types of diseases afflicting the heart. However, the electrocardiogram signals are uneven in nature and occur erratically at different time intervals during a day. Thus there arises the need for continuous monitoring of the ECG signals. The ECG signals are generally complex in nature. Hence there is a possibility of the analyst missing clinically important information which can be critical in determining the nature of the disease. Thus computer based automated analysis is recommended for early and accurate diagnosis [3].

The main challenge faced by the models for automatic heart beat classification is the variableness of the ECG waveforms from one patient to another even within the same person. However, different types of arrhythmias have certain characteristics which are universally common among all the patients. Thus the main objective of a heart beat classifier is to identify those characteristics so that the diagnosis can be general and as reliable as possible.

\subsection{The ECG Signal}

The electrocardiogram (ECG) is a real-time on-line graphical display of the electrical activity of the heart. Many electrical and mechanical abnormalities of the heart can be discovered by measuring ECG, which are potentials measured on the body surface. By extracting the ECG signal through the technique of placing surface electrodes on the limbs or chest, we can conclude the electrical sign of the contractile activity of the heart [4]. The ECG is perhaps the most commonly known, recognized, and used biomedical signals. The rhythm of the heart in term of beats per minute (bpm) may be easily estimated by counting the readily identifiable wave. More important is the fact that the ECG wave shape is altered by cardiovascular diseases such as myocardial ischemia and infarction, ventricular hypertrophy, and conduction problem [5].

Electrocardiogram for the diagnosis can be acquired clinically by the following three techniques. They are:

Standard clinical ECG(12 leads)

VCG(3 orthogonal leads)

Monitoring ECG (1 or 2 leads)

Fig .1 shows a typical heartbeat of a normal monitoring ECG signal. A heartbeat of ECG signal consists of $\mathrm{P}, \mathrm{Q}, \mathrm{R}, \mathrm{S}, \mathrm{T}$ and U waves.

The electrocardiogram is a time-varying signal that measures the electrical activity (on the surface of the human body) of the heart. The standard parameters of the ECG waveform are the $\mathrm{P}$ wave, the QRS complex and the T wave. But most of the information lies around the R peak. Additionally a small $\mathrm{U}$ wave (with an uncertain origin) is occasionally present. The cardiac cycle begins with the $\mathrm{P}$ wave, which corresponds to the period of atrial depolarization in the heart. This is followed by the QRS complex, which is usually the most relevant (recognizable) feature of an ECG waveform. The T wave follows the QRS complex and corresponds to the period of ventricular repolarisation. The ending point of the $\mathrm{T}$ wave represents the end of the cardiac cycle (presuming the absence of $U$ wave). The durations (time between the onset and offset) of particular parameters of the ECG (referred as an time interval) is of great importance since it provides a measure of the state of the heart and can show the presence of certain cardiological conditions. In practice, interval measurements, wave interpretations are carried out manually by ECG 
specialists, which may sometimes lead to human errors causing catastrophic effects.

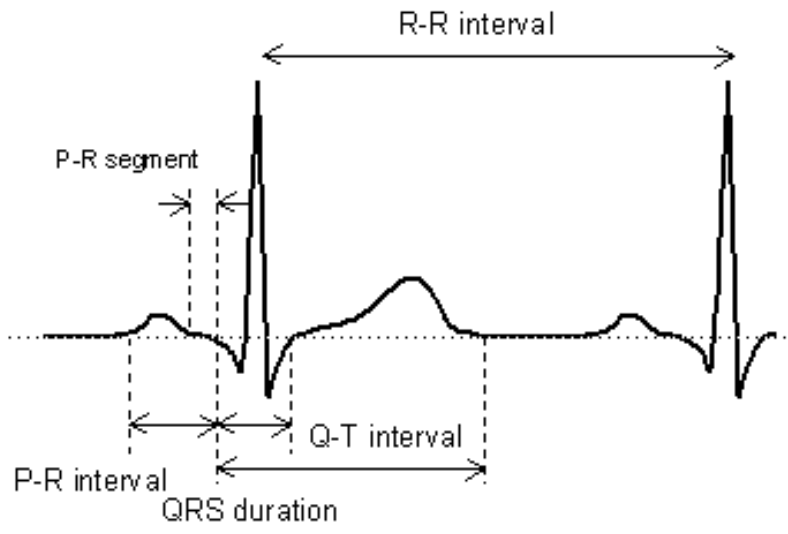

Fig. 1 A typical heart beat of ECG signal

\subsection{Detection of arrhythmia from ECG Signal}

Classification of ECG signal is an important area in biomedical signal processing. Heart arrhythmias result from any disturbance in the rate regularity and site of origin or conduction of the cardiac electric impulse [6]. Detection of these arrhythmias is well investigated and successful detectors have been designed with high sensitivity and specificity. Many arrhythmias are noticeable as sequences of heartbeats with unusual temporal features or ECG morphology.

An important step towards identifying an arrhythmia is the feature extraction from the ECG. The rhythm of the ECG signal can then be understood by knowing the features extracted from ECG. Several algorithms have been developed for automated feature extraction from ECG beats and identifying the arrhythmias. These techniques use a variety of features to represent the ECG and a number of feature extraction techniques.

Some of the Features include template matching, ECG morphology, heartbeat interval features, Wavelet transform, Daubechies Wavelets transform, ECG obfuscation method, hidden Markov models, Slope Vector Waveform (SVW) \& cepstrum coefficient method[7]. Most popular Classifier methods employed include linear discriminates, SVM, Gaussian mixture model-based, rule-based rough-set decision system, Bi-spectral analysis technique, supervised classifiers, Hermite functions and self-organizing maps [9].

The main focus of the paper is to utilize wavelet analysis methods for extracting various features like R peak, QRS complex detection \& ST segment elevation from the ECG signal for identifying myocardial Ischemia. The next section deals with ECG data analysis and fundamentals of Wavelet analysis.

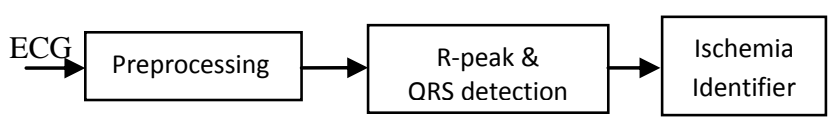

Fig. 2 Proposed system for Myocardial Ischemia detection

\section{Feature extraction}

The ECG feature extraction system provides fundamental features to be used in subsequent automatic analysis. It is therefore important to accurately extract features of prime importance: P-wave, QRS-complex, T-wave, P-Q interval, S$\mathrm{T}$ segment, and Q-T interval [5]. Designing an algorithm for the detection of these features is a difficult problem due to the time varying morphology of the signal subject to physiological conditions. Moreover the localization of wave onsets and ends is much more difficult, as the signal amplitude is low at the wave boundaries and the noise level can be higher than the signal itself. Wavelet Transform (WT) has been proposed as an alternative way to analyse the nonstationary biomedical signals, which expands the signal onto the basis functions. The WT acts as a mathematical microscope in which we can observe different parts of the signal by just adjusting the focus [6].

\subsection{Wavelet transform}

Wavelet transforms allow a signal to be decomposed such that frequency characteristics and the location of particular features in a time series may be highlighted simultaneously. In contrast to conventional techniques, the WT provides a new dimension to signal processing and event detection. Due to its time-frequency localization properties, the WT is an efficient tool for analysing non-stationary ECG signals. The Wavelet technique provides a description of a signal in a timescale domain, analogous to a time-frequency domain, allowing the representation of temporal features at multiple resolutions. This is achieve by the decomposition of the signal over dilated (scale) and translated (time) versions of a prototype wavelet. In its continuous form, the WT of a signal $\mathrm{x}(\mathrm{t})$ is defined as below-

$C W T_{c} \quad x(b)=\int_{-\infty}^{+\infty} x(t) \frac{1}{\sqrt{a}} \psi^{*}\left(\frac{t-b}{a}\right) d t$

Where (t) is the prototype (or mother wavelet), $a$ is the scale factor $\left(\mathrm{a} \in \mathrm{R}^{+}\right), \mathrm{b}$ is the translation factor $(\mathrm{b} \in \mathrm{R})$, CWT the continuous wavelet transform operator and $*$ the complex conjugate operator. The wavelet transform in equation (1), can be rewritten as a convolution product between the signal and the scaled wavelets and can also be interpreted as a filtering of the signal by band pass filters whose centre frequencies and bandwidths depend on the scaling factor. High scales translate into long, slow wavelets equivalent to narrow, low-frequency filters, while lower scales produce shorter, faster wavelets equivalent to wider, higher-frequency filters. With these properties, WT achieves an ideal balance of time and frequency resolution: slow trends are represented with a high frequency resolution and a low time resolution, while fast components are well defined in time but less in frequency.

Such inherent multiresolution capabilities make WTs very effective at detecting and representing singularities, and WTs have been applied in many occasions to ECG analysis. The ECG signals are considered as representative signals of cardiac physiology, useful in diagnosing cardiac disorders. The ECG signal, consisting of many data points, can be compressed into a few parameters by the WT. These parameters characterize the behaviour of the ECG signal and they can be used for recognition and diagnostic purposes. 


\subsection{Daubechies Wavelets}

Daubechies wavelets are the most popular wavelets among various wavelet families found in the literature. The Daubechies wavelets are used in versatile applications. The wavelets filters are selected based on their ability to analyse the signal and their shape in an application. Figure 3 shows nine members of the Daubechies family.

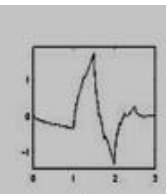

$\mathrm{db} 2$

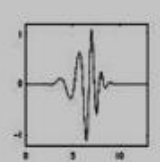

$\mathrm{db} 7$

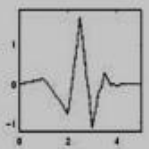

db3

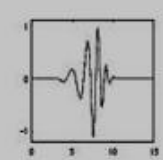

$\mathrm{db} 8$

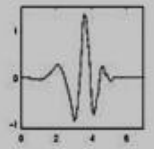

$\mathrm{db} 4$

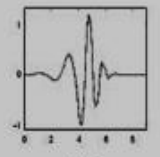

db5

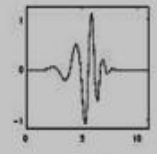

$\mathrm{db6}$
Fig 3: Nine members of the Daubechies family

The ECG signals are considered as representative signals of cardiac physiology, useful in diagnosing cardiac disorders. The most complete way to display this information is to perform spectral analysis. The wavelet transform provides very general technique which can be applied to many signal processing applications. Different features can be computed and manipulated in compressed domain using wavelet coefficients. All these means that we can apply the wavelet transform on the ECG signal and convert it to the wavelet coefficients or parameters. The obtained coefficients characterize the behaviour of the ECG signal and the number of these coefficients are small than the number of original signal. This reduction of feature space is particularly important for recognition and diagnostic purposes. [11]

\subsection{Selection of suitable wavelet}

The selection of relevant wavelet is an important task before starting the feature extraction. The choice of wavelet depends upon the type of signal to be analysed. The wavelet similar to the shape of signal is selected. There are several wavelet families like Harr, Daubechies, Biorthogonal, Coiflets, Symlets, Morlet, Mexican Hat, Meyer etc. and several othe Real and Complex wavelets. We have tried Daubechies 4 (Db4), Db6, Db8 for accurate results. However, Daubechies 4 (Db4) Wavelet has been found to give details more accurately. Due to its symmetry with a QRS complex as well as concentration of energy spectrum at lower frequencies, Daubechies wavelets are utilized. Number of constant terms for Daubechies wavelets was decided between 4 or 6 . After many trials, $\mathrm{Db} 4$ was chosen as it provided excellent results.

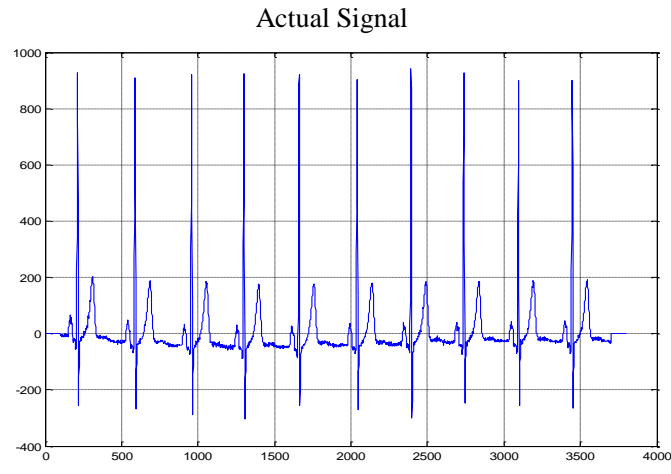

Fig.4: Actual signal acquired from record 303 of MITBIH data base

Denoised and smoothed signal

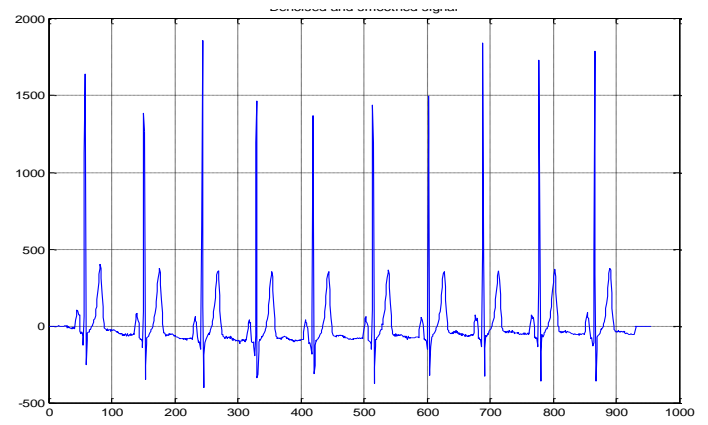

Fig.5: Denoised and smoothed ECG signal
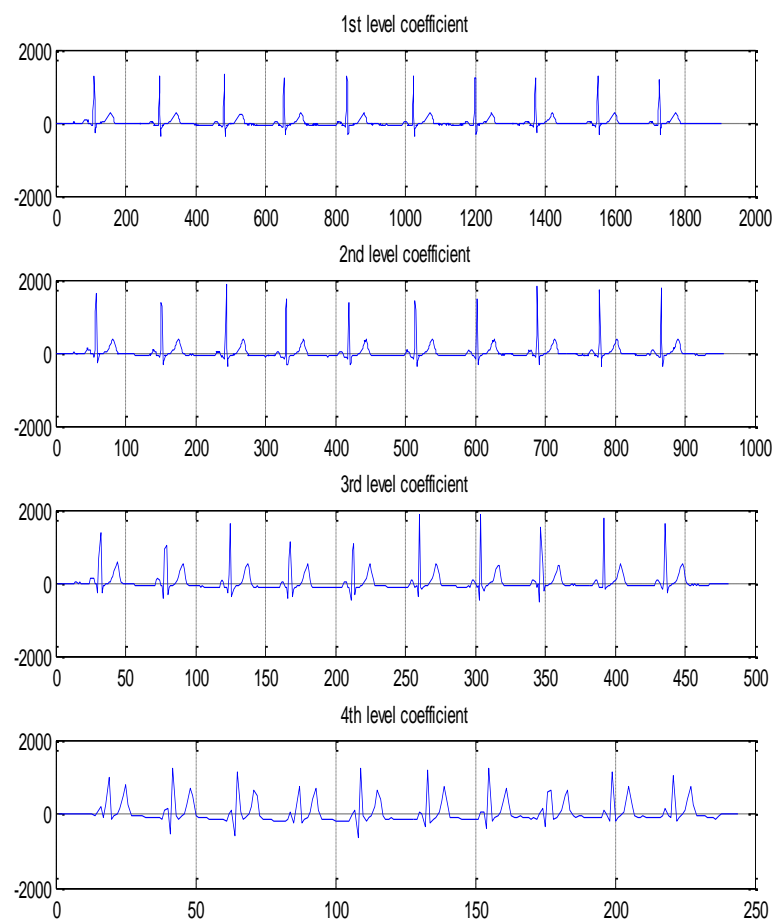

Fig. 6: Plot of Daubechies coefficients upto $4^{\text {th }}$ level 
Then, Wavelet decomposition of the ECG samples is performed up to four levels which results in the samples at much lower frequency than the original signal. Thus, QRS complex is preserved. Further, extracting and then plotting the coefficients at each level result in the plot of cleaner signal. Figure 6 shows the plot of coefficients of the decomposed data of a Myocardial Ischemia diseased person. It is clear from the figure that $4^{\text {th }}$ level decomposed data is noise free. Therefore, we consider this signal as ideal ECG signal from which all the peaks are detected.

\section{METHODOLOGY \& DATA ANALYSIS}

The need of the feature extraction process is to extract diagnostically important information from the ECG signal. The algorithm is developed for myocardial ischemia subclass of arrhythmia whose characteristic conditions were collected. Algorithm presented can be applied directly at one run over the whole digitized ECG signal. MIT-BIH database was utilized to validate the algorithm.

\subsection{Ischemic event feature extraction algorithm}

1. ECG data of short length (about 25 QRS complex) is taken.

2. Wavelet Decomposition is carried out to down sample the signal for taking the samples at much lower frequency than the original signal.

3. Extracted the Daubechies wavelets coefficients upto $4^{\text {th }}$ level and coefficients are plotted to get smooth and cleaner signals

4. $\mathrm{R}$ peaks are detected in the down sampled signal by finding the maximum value of voltage in particular range of time.

5. The detected $\mathrm{R}$ peaks are mapped in to down sampled signal to get $\mathrm{R}$ peaks in Original signal.

6. $\mathrm{P}, \mathrm{Q}, \mathrm{S}, \mathrm{T}$ peaks are detected by taking the reference of $\mathrm{R}$ peaks.

7. ST segment is calculated from S-Offset and T-Onset.

Fig.7: Detection of $P, Q, R, S \& T$ points on ECG

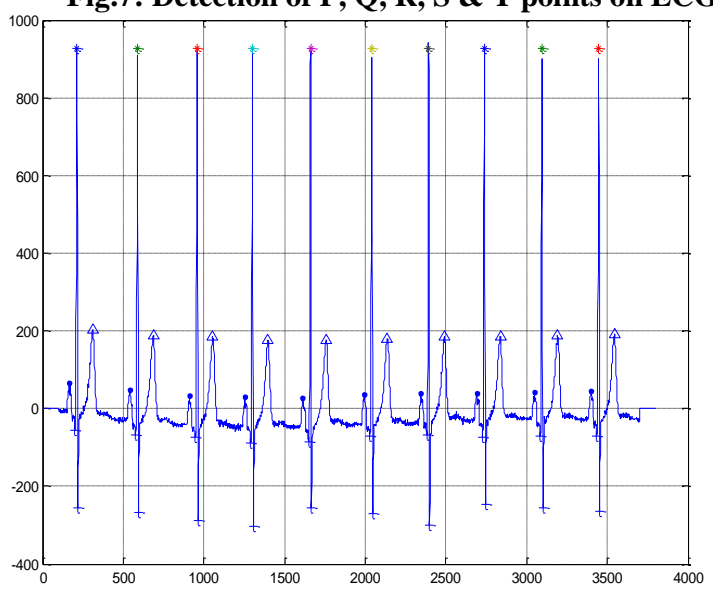

\section{RESULTS \& DISCUSSIONS:}

Table 1 shows detection Results on the randomly selected files of MIT-BIH database:

\begin{tabular}{|l|l|l|l|l|}
\hline $\begin{array}{l}\text { Record } \\
\text { Number }\end{array}$ & $\begin{array}{l}\text { Total } \\
\text { beats } \\
\text { tested }\end{array}$ & $\begin{array}{l}\text { Beats } \\
\text { correctly } \\
\text { recognized }\end{array}$ & $\begin{array}{l}\text { Recognition } \\
\text { Accuracy } \\
\text { in \% }\end{array}$ & $\begin{array}{l}\text { Arrhythmia } \\
\text { Detected }\end{array}$ \\
\hline 300 & 93 & 92 & 98.9247 & MI \\
\hline 301 & 57 & 57 & 100 & MI \\
\hline 302 & 64 & 61 & 95.3125 & MI \\
\hline 303 & 87 & 86 & 98.8505 & $\begin{array}{l}\text { MI with T } \\
\text { inverted } \\
\text { logic }\end{array}$ \\
\hline 304 & 54 & 54 & 100 & $\begin{array}{l}\text { MI with T } \\
\text { inverted } \\
\text { logic }\end{array}$ \\
\hline 306 & 64 & 64 & 100 & $\begin{array}{l}\text { MI with T } \\
\text { inverted } \\
\text { logic }\end{array}$ \\
\hline 310 & 94 & 94 & 100 & MI \\
\hline 312 & 61 & 59 & 96.7213 & MI \\
\hline 313 & 80 & 79 & 98.75 & MI \\
\hline 314 & 66 & 66 & 100 & $\begin{array}{l}\text { MI with T } \\
\text { inverted } \\
\text { logic }\end{array}$ \\
\hline Average & 720 & 712 & 98.8888 & \\
\hline
\end{tabular}

An algorithm for detection of R peaks and QRS complex has been developed using Daubechies wavelts. Table 1 shows the detection results on the randomly selected files of MITBIH database. The information about $\mathrm{R}$ peak and $\mathrm{QRS}$ complex obtained is very useful for ECG classification, analysis, diagnosis, authentication and performance identification of performance. The ST segment elevation and inverted T peak detected in the QRS complex is used for determination of myocardial Ischemia class of arrhythmias. This information can also serve as an input to a system that allows automatic cardiac diagnosis. Also, the physiological and geometric differences of the heart in different individuals display certain uniqueness in their ECG signals which can be used as a tool for Identification and verifications of individuals. Detection accuracy of $98.88 \%$ is achieved through this algorithm which is a promising result.

\section{Conclusion}

By using the wavelet transform, we are able to detect various features from an ECG signal. Since it was difficult to avail subjects with positive arrhythmia, the algorithm was tested on the already available database. The algorithm created in MATLAB was successful in eliminating noises and baseline drifts that could degrade the accuracy of the algorithm. We also succeeded in extracting all the events of ECG signal. The QRS complex was detected and we used to detect inverted T peak and ST segment elevation was calculated. The application of Daubechies wavelet transform speeds the signal processing of ECG which gives us faster response. In a view to solve the problems related to the patients regularly using ECG signal, this work proposes a better method to detect symptoms of some major arrhythmias. 


\section{ACKNOWLEDGMENTS}

Our thanks to the experts who have contributed in development of MIT-BIH database.

\section{REFERENCES:}

[1] Features Extraction of ECG signal for Detection of Cardiac arrhythmias by P.D.Khandait, N.G.Bawane, S.S.Limaye: National Conference on Innovative paradigms in Engineering \& Technology (NCIPET2012), proceedings published by International Journal of Computer Applications (IJCA)

[2] Ebrahimzadeh, A., A. Khazaee, and V. Ranaee. Classification of the electrocardiogram signals using supervised classifiers and efficient features. Comput. Methods Programs Biomed. 99:179-194, 2010.

[3] Acharya, U. R., M. Sankaranarayanan, J. Nayak, C. Xiang, and T. Tamura. Automatic identification of cardiac health using modeling techniques: a comparative study. Inform. Sci. 178:4571-4582, 2008.

[4] Alan Jovic, and Nikola Bogunovic, "Feature Extraction for ECG Time- Series Mining based on Chaos Theory," Proceedings of 29th International Conference on Information Technology Interfaces, 2007.

[5] V. S. Chouhan, and S. S. Mehta, "Detection of QRS Complexes in 12- lead ECG using Adaptive Quantized Threshold," IJCSNS International Journal of Computer Science and Network Security, vol. 8, no. 1, 2008.

[6] Yu, S. N., and K. T. Chou. Selection of significant for ECG beat classification. Expert Syst. Appl. 36:20882096, 2009.
[7] Kuo-Kuang Jen, and Yean-Ren Hwang, "ECG Feature Extraction and Classification Using Cepstrum and Neural Networks," Journal of Medical and Biological Engineering, vol. 28, no. 1, 2008.

[8] Ebrahimzadeh, A., A. Khazaee, and V. Ranaee. Classification of the electrocardiogram signals using supervised classifiers and efficient features. Comput. Methods Programs Biomed. 99:179-194, 2010.

[9] Khadra, L., A. S. Al-Fahoum, and S. Binajjaj. A quantitative analysis approach for cardiac arrhythmia classification using higher order spectral techniques. IEEE Trans. Biomed. Eng. 52:1840-1845, 2005.

[10] N.V.Thakor, J.G.Webster and W.J.Tompkins, "Estimation of QRS complex power spectra for design of a QRS filter", IEEE Transactions on Biomedical Engineering, vol. BME-31, no. 11,pp. 702-706, 1986, pp. 702-706, Nov, 1986

[11] E.D. Ubeyli, (2008) "Implementing wavelet transform / mixture of experts network for analysis of ECG beats", Expert system Vol.25,pp.150-162.

[12] Robert J. Huzar, Basic Dysrhythmias, Interpretation and Management (C.V.Mosby Co., 1988).

[13] Goldberg AL, Amaral LAN, Glass L, Hausdorff JM, Ivanov PCh, Mark RG, Mietus JE, Moody GB, Peng CK, Stanley HE. PhysioBank, PhysioToolkit and PhysioNet: Components of a New Research Resource for Complex Physological Sirnals. Circulation 101(23):e215e220 